\title{
Komplikationen durch Nahrungsergänzungsmittel
}

\section{Die Annahme, dass Nahrungsergän- zungsmittel, wenn schon nicht nützen, so doch zumindest nicht schaden, ist offenbar falsch. Einer US-Studie zu- folge verursachen sie eine hohe Zahl von Notaufnahmen.}

Die Studienautoren um Andrew Geller vom Center for Disease Control and Prevention in Atlanta fanden in den Berichten von 63 Notaufnahmeabteilungen insgesamt 3.667 Fälle, die in den Jahren von 2004 bis 2013 mit unerwünschten Wirkungen von Nahrungsergänzungsmitteln in Zusammenhang ge- bracht worden waren. Auf Basis dieser Stichprobe errechneten sie, dass in den USA durchschnittlich etwa 23.000 Notaufnahmen pro Jahr wegen solcher Nebenwirkungen passieren. Diese würden ihren Schätzungen zufolge jährlich zu etwa 2.154 Hospitalisierungen führen.

Erstaunlich häufig, nämlich in einem Viertel der untersuchten Fälle, waren junge Menschen im Alter zwischen 20 und 34 Jahren betroffen. Bei etwa einem Fünftel der Notaufnahmen hatte ein Kind ein Präparat - meist einen Mikronährstoff $(61,9 \%)$ - unbeaufsichtigt zu sich genommen.
Auffällig häufig und vor allem bei jungen, 20- bis 35-jährigen Personen kam es unter den sogenannten Schlankheitspillen zu kardialen Beschwerden wie Herzrasen, Brustschmerzen oder Tachykardie; diese Präparate waren für $71,8 \%$ der mit solchen Beschwerden einhergehenden Notaufnahmen verantwortlich. Ebenfalls nicht selten traten kardiale Beschwerden in Verbindung mit Bodybuilding-Produkten und sexualfördernden Substanzen auf (in 49,8\% und $37,3 \%$ ).

- Geller Al et al. N Engl J Med 2015;373:1531-40

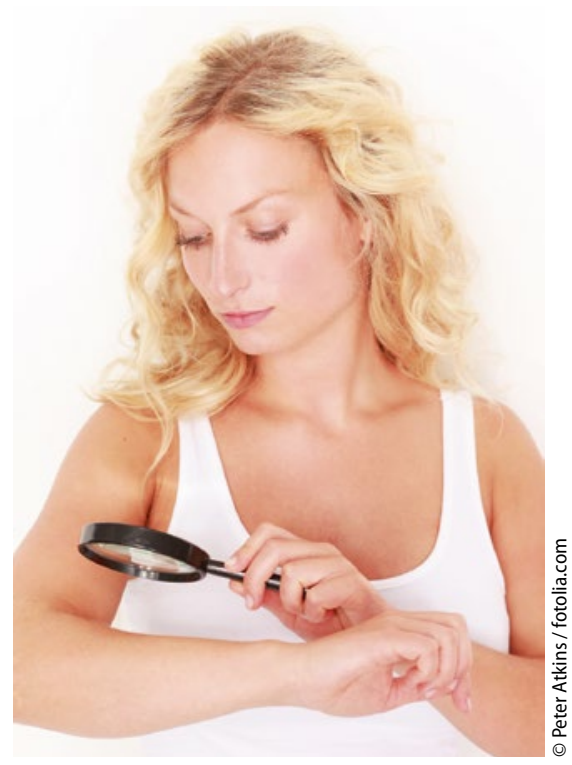

Marker für das Melanomrisiko: die Zahl der Nävi auf dem rechten Arm.
Wahrscheinlichkeit, an einem Melanom zu erkranken, abschätzen. Dadurch ließen sich möglicherweise mehr Patienten als bisher mit einem erhöhten Risiko identifizieren.

- Ribero S et al. Br J Dermatol 2015; online 19. Oktober signifikante Korrelation - wenn auch weniger stark - stellten die Ärzte zwischen der Zahl der Nävi an den Beinen und auf dem Rücken und der Gesamtzahl der Nävi fest.

Nach Ansicht der britischen Ärzte lässt sich in der Hausarztpraxis anhand der auf dem rechten Arm gezählten Nävi rasch die Gesamtzahl und damit die

Der beste Vorhersagewert wurde für den rechten Arm ermittelt: Fanden sich der Gesamtkörperoberfläche zu haben, neunfach erhöht (adjustierte Odds Ratio 9,38; 95\%-Konfidenzintervall 6,71 bis 13,11). Vergleichsgruppe waren Frauen mit weniger als elf Nävi auf dem rechten rm.

ach lanomrisiko „ab einer Zahl von 50 Nävi etwa um den Faktor 4 bis 5, ab 100 melanozytären Nävi auf das 8- bis 10-Fache ten deshalb in einer Zwillingsstudie welcher Körperregion die Zahl der Nävi am besten mit dem Melanomrisiko korreliert. Zwischen 1995 und 2003 wurden bei allen Teilnehmerinnen der Hauttyp, die Haar- und Augenfarbe sowie Sommersprossen und Muttermale in 17 verschiedenen Regionen dokumentiert. 